\title{
ON THE POLYNOMIALS WHOSE DERIVATIVES HAVE INTEGRAL VALUES AT THE INTEGERS
}

\author{
ERNST G. STRAUS
}

It is well known that the polynomials, which together with their derivatives have integral values at a fixed point $x=x_{0}$, have the basis

$$
\left(x-x_{0}\right)^{n} / n !, \quad n=0,1,2, \cdots,
$$

over the integers. That is to say every polynomial with that property is of the form $\sum_{k=0}^{n} c_{k}\left(x-x_{0}\right)^{k} / k$ ! where $c_{0}, c_{1}, \cdots, c_{n}$ are integers. Similarly it is known that a basis over the integers for those polynomials whose values are integral at all integers is given by

$$
1, x(x-1) \cdots(x-n+1) / n !, \quad n=1,2, \cdots \text {. }
$$

It is the purpose of this note to construct a basis over the integers for the polynomials which together with all their derivatives have integral values at all integers. We shall call such polynomials $I$ polynomials.

In order to construct this basis we prove a few introductory lemmas:

LEMMA 1. Let $D_{n}$ be the greatest integer such that there exists an I-polynomial of the form

$$
f_{n}(x)=x^{n} / D_{n}+\text { lower powers, }
$$

and let

$$
f(x)=(a / b) x^{n}+\text { lower powers, } \quad(a, b)=1,
$$

be any I-polynomial; then $b \mid D_{n}$.

Proof. Let $m$ be the least common multiple of $D_{n}$ and $b$ and let $k_{1}=m / D_{n}, k_{2}=m / b$; then $\left(k_{1}, k_{2}\right)=\left(k_{1}, a\right)=1$. Hence there exist two integers $c_{1}, c_{2}$ such that $c_{1} k_{1}+c_{2} k_{2} a=1$ and $c_{1} f_{n}(x)+c_{2} f(x)=x^{n} / m+$ lower powers.

From the definition of $D_{n}$ it follows that $m \leqq D_{n}$; but since $m$ is a multiple of $D_{n}$ we have $m=D_{n}$ or $b \mid D_{n}$.

LEMma 2. The I-polynomials

$$
f_{n}(x)=x^{n} / D_{n}+\text { lower powers, } \quad n=0,1,2, \cdots,
$$

where $D_{n}$ is as in Lemma 1, form a basis over the integers of the I-polynomials.

Received by the editors January 26, 1950. 
Proof. We proceed by induction. The lemma is obviously true for polynomials of degree 0 . Assume the lemma true for all polynomials of degree $<n$. Let

$$
f(x)=(a / b) x^{n}+\text { lower powers }
$$

be an $I$-polynomial; then according to Lemma $1, c_{n}=a D_{n} / b$ is an integer. Thus $f(x)-c_{n} f_{n}(x)$ is an $I$-polynomial of degree $<n$ and

$$
\begin{aligned}
& f(x)-c_{n} f_{n}(x)=c_{0} f_{0}(x)+\cdots+c_{n-1} f_{n-1}(x) \\
& \text { or } f(x)=c_{0} f_{0}(x)+\cdots+c_{n} f_{n}(x) .
\end{aligned}
$$

Lemma 3. If $m<n$, then $D_{m} \mid D_{n}$.

Proof. If $f_{m}(x)=x^{m} / D_{m}+\cdots$ is an $I$-polynomial, then so is $x^{n-m} f_{m}(x)=x^{n} / D_{m}+\cdots$ and hence according to Lemma 1 we have $D_{m} \mid D_{n}$

Lemma 4. If $p$ is a prime and $p^{k} \mid D_{n}$, then $p^{k} \mid[n / p] !$ or, in other words,

$$
k \leqq\left[n / p^{2}\right]+\left[n / p^{3}\right]+\cdots .
$$

Proof. Let $f(x)$ be a polynomial with integral coefficients of least degree such that $f(x) / p^{k}$ is an $I$-polynomial, and let $j$ be any one of the numbers $0,1, \cdots, p-1$. Let $m$ be the multiplicity of $j$ as a root of the congruence $f(x) \equiv 0(\bmod p)$, that is to say

$$
f(x)=(x-j)^{m} g(x)+p h(x)
$$

where $g(x), h(x)$ are polynomials with integral coefficients and $g(j)$ $\not \equiv 0(\bmod p)$.

Differentiating $m$ times we obtain

$$
f^{(m)}(x)=m ! g(x)+(x-j) k(x)+p h^{(m)}(x)
$$

where $k(x)$ is a polynomial with integral coefficients. We have $h^{(m)}(x)$ $=m ! l(x)$ where $l(x)$ has integral coefficients and hence:

$$
f^{(m)}(x)=m ! g(x)+(x-j) k(x)+p m ! l(x) .
$$

Let $p^{r}$ be the highest power of $p$ which divides $m$ !; then we obtain

$$
f^{(m)}(j) \equiv m ! g(j)\left(\bmod p^{r+1}\right)
$$

and hence unless $r \geqq k$ we cannot have $f^{(m)}(j) / p^{k}=$ integer.

Since $j$ was chosen as any one of the numbers $0,1, \cdots, p-1$ we see that, $\bmod p, f(x)$ must be divisible by $x^{m}(x-1)^{m} \cdots(x-p+1)^{m}$, where $m$ is such that $p^{k} \mid m$ !. But the degree of $f(x)$ is then greater than or equal to $p m$. 
If we introduce the symbol $(n)_{p}$ for the greatest exponent, $m$, such that $p^{m} \mid n$, then we can state

Lemma 5. $D_{n} \leqq \prod_{p-\text { prime }} p^{([n / p] 1)_{p}}=n ! / \prod_{p=\text { prime }} p^{[n / p]}$.

The proof is an immediate consequence of Lemmas 3 and 4.

We now can prove the following theorem.

THEOREM. The polynomials

$$
f_{n}(x)=\prod_{\left.p^{[n / p}\right] x(x-1) \cdots(x-n+1) / n !}
$$

form a basis over the integers of the I-polynomials.

Proof. According to Lemmas 5 and 2 it suffices to show that the $f_{n}(x)$ are $I$-polynomials. Since $f_{n}(x)$ is an integral multiple of $x(x-1) \cdots(x-n+1) / n !$, it is integral-valued. We now proceed by induction and show that if $f_{k}(x)$ is an $I$-polynomial for $k<n$, then $f_{n}^{\prime}(x)$, and hence $f_{n}(x)$, is an $I$-polynomial.

We have

$$
f_{n}^{\prime}(x)=\frac{\prod p^{[n / p]}}{n !} \sum_{i=0}^{n-1} \frac{x(x-1) \cdots(x-n+1)}{x-i}
$$

and since

$$
\begin{aligned}
& \frac{x(x-1) \cdots(x-n+1)}{x-i} \\
& =\sum_{k=0}^{n-i-1}(-1)^{k} \frac{(n-i-1) !}{(n-i-k-1) !} x(x-1) \cdots(x-n+2+k)
\end{aligned}
$$

we have

$$
\begin{aligned}
\sum_{i=0}^{n-1} & \frac{x(x-1) \cdots(x-n+1)}{x-i} \\
& =\sum_{k=0}^{n-1}(-1)^{k} \frac{n(n-1) \cdots(n-k)}{k+1} x(x-1) \cdots(x-n+2+k)
\end{aligned}
$$

or

$$
\begin{aligned}
f_{n}^{\prime}(x) & =\prod p^{[n / p]} \sum_{k=1}^{n}(-1)^{k+1} \frac{1}{k(n-k) !} x(x-1) \cdots(x-n+k+1) \\
& =\sum_{k=1}^{n}(-1)^{k+1} \frac{1}{k} \prod p^{[n / p]-[(n-k) / p]} f_{n-k}(x) .
\end{aligned}
$$

We now show that 


$$
\prod_{p=\text { prime }} p^{[n / p]-[(n-k) / p] / k}=A_{k}
$$

is an integer. $A_{k}$ is certainly an integer modulo $p$ if $k$ is not divisible by $p$. If $k$ is divisible by $p$ then $[(n-k) / p]=[n / p]-k / p$ and

$$
p^{[n / p]-[(n-k) / p]}=p^{k / p}
$$

and since obviously

$$
k / p \geqq(k)_{p}
$$

we have again that $A_{k}$ is an integer modulo $p$. Thus $A_{k}$ is an integer for

$$
k=1, \cdots, n-1 ; \text { and } f_{n}^{\prime}(x)=\sum_{k=1}^{n}(-1)^{k+1} A_{k} f_{n-k}(x)
$$

is an $I$-polynomial.

In conclusion we should like to pose a number of problems:

(1) Instead of considering those polynomials whose values at the rational integers are rational integers, we consider the polynomials whose values at the algebraic integers of a certain field are algebraic integers of the same field. Find a basis for the polynomials which are integral-valued in this generalized sense, and for the polynomials which together with all their derivatives are integral-valued in the generalized sense. It may be conjectured that there exist bases of the form $\left(\alpha_{n} / n !\right) x(x-1) \cdots(x-n+1)$ where the $\alpha_{n}$ are algebraic integers.

(2) Show that the only (entire) analytic functions which have integral (Gaussian integral) derivatives at all (infinitely many) integers (Gaussian integers) are polynomials. We have shown in another paper that a transcendental entire function with integral derivatives of all orders at infinitely many integral points would have to be of infinite order.

(3) Our problem can be generalized in many other directions. For example, if instead of considering a basis for the $I$-polynomials, we look for a basis of those polynomials which have integral derivatives of all orders, not at all integral points, but only at the points $0,1, \cdots, m-1$, then we obtain a basis $f_{m n}(x) ; n=0,1,2, \cdots$ with the denominators

$$
D_{m n}=\prod_{p<m} p^{([n / p] !)_{p}} \prod_{p \geqq m} p^{([n / m] !) p}
$$

and the numerators

$$
\{x(x-1) \cdots(x-m+1)\}^{[n / m]} x(x-1) \cdots(x-n+m[n / m]+1) \text {. }
$$

University of California, Los Angeles 